\title{
Effect of Injector Wall Heat Flux on Cryogenic Injection
}

\author{
Daniel T. Banuti* and Klaus Hannemann ${ }^{\dagger}$ \\ German Aerospace Center (DLR), Institute of Aerodynamics and Flow Technology, Göttingen, Germany
}

\begin{abstract}
This paper reports on numerical investigation and discussion of a cryogenic injection test case. This is a first step towards a numerical model for high pressure liquid rocket engine injection. Experiments with nitrogen injection at supercritical pressures are an accepted way of studying flow phenomena relevant for high pressure liquid rocket engines without introducing the complexities of mixing and combustion. CFD simulations of these cryogenic injection cases are found to typically agree on predicting a region of constant high density, extending some 10 injector diameters downstream into the chamber. This is in agreement with the traditional interpretation of liquid injection where instabilities on the phase boundary grow until the jet disintegrates. However, many experiments do not show this behavior: exceeding the critical pressure of the injected fluid, phase interfaces seize to exist, the jet tends to behave more like a dense gas jet with a rapid drop off of density starting at the injector exit. It is thus unclear whether the concept of a liquid core length is still true for thermodynamic states at temperatures near the critical point and supercritical pressures. To study this, experimental and numerical boundary conditions have been analyzed. Computations have been carried out using the DLR TAU code extended by a treatment for real gas thermodynamics. It has been found that the absence of a dense core, as found in experiments, can be reproduced numerically if numerical boundary conditions are chosen appropriately: taking into account heat transfer inside the injector leads to a preheating of the cryogenic stream and the development of a distinct radial density profile. This preheated jet then shows the more realistic immediate reduction of density instead of a dense core.
\end{abstract}

\section{Introduction}

Developing high power efficient liquid rocket engines (LRE), the trend towards high pressure combustion is clearly dominating. This is certainly true for main stage engines, such as the European Vulcain at a combustion chamber pressure of $100 \mathrm{MPa}$, or the American Space Shuttle Main Engine (SSME) at almost twice this pressure level but can also be witnessed for upper stage engines (Vinci, Aestus). Increasing the chamber pressure is a way to increase both thrust and the efficiency (i.e. fuel consumption) of thrust creation.

Another trend in the development of thrust chambers is the desire to support experiments by numerical computations. This serves several purposes: 1) CFD can help designing and interpreting experiments; 2)the number of expensive and possibly dangerous experiments can be reduced; 3.) more insight is gained on the physical behavior inside the rocket combustion chamber where experimental accessibility is limited.

Typically, in methods used during the industrial design process, the flow inside the chamber is modelled as a gaseous Eulerian continuum. At the injector face, fuel injection is modelled by particle tracking of individual droplets leaving the injector. Their trajectory through and interaction with the carrier phase is integrated in a Lagrangian reference frame. This approach is hence dubbed Statistical Eulerian Lagrangian (SEL). These methods are in routine industry use today and are highly successful. ${ }^{14}$

But how does this approach compare to the physical appearance of the processes? LRE propellant injection

\footnotetext{
* Research Engineer and PhD Student, German Aerospace Center (DLR), Institute of Aerodynamics and Flow Technology, Spacecraft Section, Göttingen, Bunsenstr. 10, Germany.

${ }^{\dagger}$ Head of Spacecraft Section, German Aerospace Center (DLR), Institute of Aerodynamics and Flow Technology, Göttingen, Bunsenstr. 10, Germany, Member AIAA.
} 
phenomena have been studied by several research groups. A thorough overview of the physical process is given by Oschwald et al. ${ }^{12}$ One striking result is that the oxidizer does not leave the injector as a cloud of separate particles. Instead, the process looks more like what one is used to from a garden-hose: a compact stream of fluid leaving the injector and entering the combustion chamber. Model experiments with nitrogen and helium show a strong dependency of flow topology on the chamber pressure: at subcritical pressures, a clear separation between liquid nitrogen and gaseous helium can be observed. The jet breaks up, ligaments and droplets are formed, the effect of surface tension is clearly visible. This result remains true when nitrogen is replaced by oxygen and helium by hydrogen, as long as no reaction between both species occurs. Shadowgraphs of the reacting case still show the clear distinction between fuel and oxidizer but small structures, such as ligaments and droplets, have vanished. Apparently, small portions of liquid phase are consumed rapidly in a high temperature environment.

The topology for the nitrogen/helium case changes drastically upon an increase in pressure enough to exceed the nitrogen's critical pressure. The center jet cannot be distinguished from the surrounding jet any longer, sharp lines diffuse, a gradual change in density through the flow field prevails. Further, with no surface tension acting, the dense fluid is no longer confined to spherical shapes. It is concluded that the whole process looks more like mixing than like atomization. Furthermore, the cryogenic jet no longer behaves like an ideal gas, instead, real gas equations of state have to be taken into account. Figure 1(a) compares the ideal gas equation of state and real fluid data of nitrogen taken from the NIST database. It can be seen that for temperatures higher than $300 \mathrm{~K}$ and pressures between 4 and $6 \mathrm{MPa}$ no difference can be observed in the density between real gas and ideal gas. Moving to cryogenic temperatures causes a massive deviation: while at supercritical pressures no condensation occurs, still a massive, albeit continuous, increase in density can be observed in real gas behavior. Below $100 \mathrm{~K}$, a quasi incompressible domain is entered where the fluid density no longer depends upon the pressure. Figure 1(b) compares this to data of oxygen and hydrogen at $6 \mathrm{MPa}$, as present in a typical single injector combustion chamber. ${ }^{6}$

Thus, in both supercritical and subcritical regimes, the oxidizer (or its model fluid nitrogen) does not exist as a dilute cloud of individual droplets, moving through the fuel (or its model fluid helium) carrier phase. Instead, the oxidizer is existing as a continuous stream - at subcritical pressures with a sharp interface, at supercritical pressures with a diffuse interface. The phenomenological justification for SEL models is hence no longer given, even though they are still applied to this regime with great success. Nonetheless, this is motivation enough to investigate a possible alternative model which takes into account the phenomenological physical behavior. In accordance with experimental findings, an approach is chosen where the non particulate character of rocket engine fuel injection is emphasized, namely a Eulerian representation of both oxidizer and fuel. These Statistical Eulerian Eulerian (SEE) models have gained popularity in recent years $\left({ }^{8,13}\right)$. The corresponding multiphase two-fluid model for subcritical pressures has been discussed by Banuti and Hannemann. ${ }^{1}$ Here, the emphasis is placed on the single phase model. The single phase model is based on the assumption that supercritical atomization can be modelled using a mixing assumption.

In this paper, as a first step, a pure substance test case is being investigated: injection of cryogenic nitrogen into a high pressure nitrogen environment at room temperature. It is important to understand this case before progressing to more complex models. Thus, the question is, whether we are able to reproduce characteristic features of this category of flow, which are identified by briefly reviewing experiments and CFD simulations. Particularly the occurrence of a 'liquid core' is studied.

\section{A Test Case for Cryogenic Injection}

\section{II.A. The Experiment}

While publications exist about several cryogenic injection experiments, the most thorough campaign appears to have been carried out by Mayer et al. ${ }^{11}$ and Branam et al. ${ }^{3}$ In their experiments, cryogenic nitrogen at supercritical pressures is injected into a chamber filled with nitrogen at supercritical temperature. Axial and radial density profiles, core length, jet spreading angles, length scales and shadowgraphs are published for $4 \mathrm{MPa}, 5 \mathrm{MPa}$, and $6 \mathrm{MPa}$ chamber pressure, $100 \mathrm{~K}, 120 \mathrm{~K}$, and $130 \mathrm{~K}$ fluid temperature at $5 \mathrm{~m} / \mathrm{s}$ and $2 \mathrm{~m} / \mathrm{s}$ injection velocity.

The test chamber consists of a circular cylinder with $122 \mathrm{~mm}$ diameter and $1 \mathrm{~m}$ length. The cylinder wall is heated to $298 \mathrm{~K}$, the injector and the faceplate are not heated. Nitrogen is being injected at the center of the faceplate through a cylindrical injector with $2.2 \mathrm{~mm}$ diameter. The injector is $90 \mathrm{~mm}$ long, it is preceded by a $6 \mathrm{~mm}$ diameter tube. The pressure in the chamber is controlled via a pressure valve. 


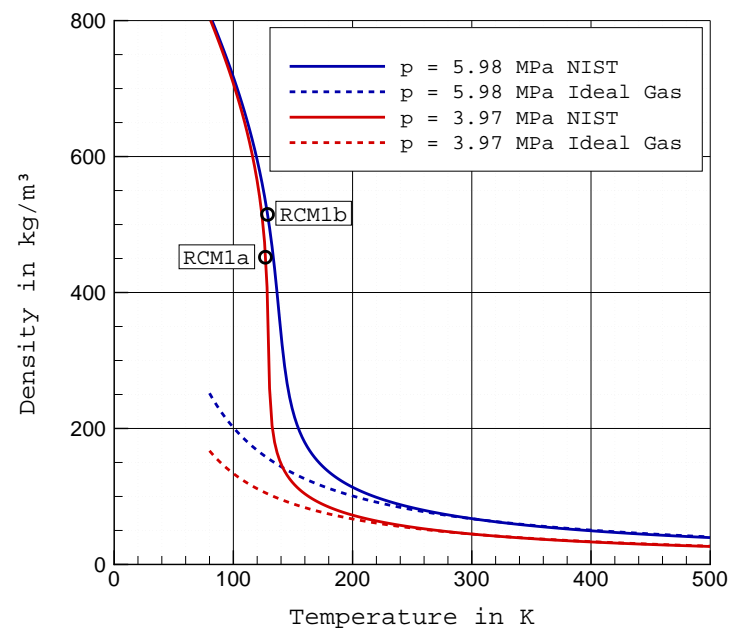

(a) Nitrogen isobaric density-temperature plot.

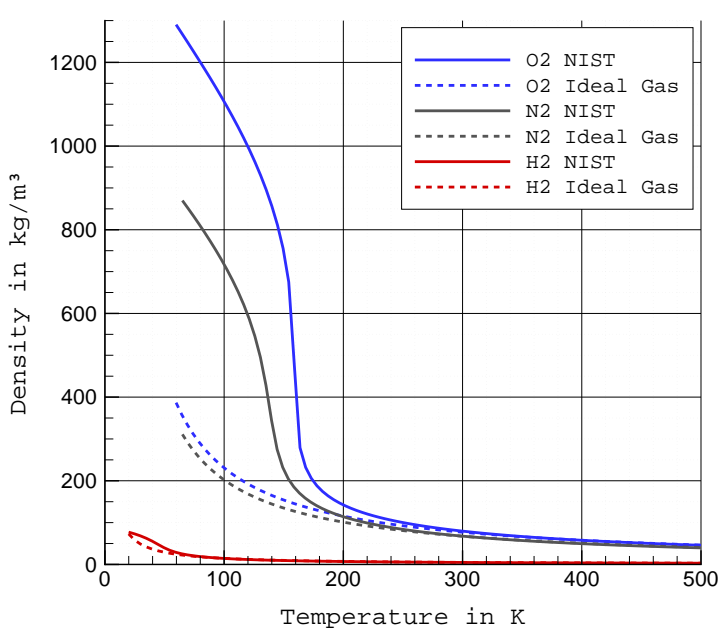

(b) Density-temperature plot at $6 \mathrm{MPa}$.

Figure 1. Effects of real gas equation of state.

The experimental procedure is as follows. The chamber is pressurized with nitrogen at 298 K. Cryogenic nitrogen is fed through the injector, the mass flux is controlled. The temperature is measured immediately in front of the convergent part of the injector, i.e. $90 \mathrm{~mm}$ upstream of the injector exit. The cryogenic nitrogen cools the structure of the injector. When the measured temperature $\mathrm{T} 1$ reaches the nominal temperature of the experiment, the Raman density measurements are carried out. In order to measure the temperature of the injected fluid, a subsequent experiment is carried out in the very same manner, except that instead of the Raman measurement, a thermocouple of $1 \mathrm{~mm}$ diameter is introduced to the free jet in two positions: axially, reaching $1 \mathrm{~mm}$ inside the injector $\left(\mathrm{Tb}_{i n j}\right)$; and radially, $1 \mathrm{~mm}$ downstream of the injector exit $\left(\mathrm{Ta}_{i n j}\right)$. Obtained axial density distributions are shown in Fig. 2(a).

Conditions of the test cases are compiled in Tab. 1, case numbering follows Mayer.

Table 1. Overview of test cases from Mayer et al., ${ }^{11} \mathrm{~T} 1$ from Branam et al. ${ }^{3}$

\begin{tabular}{rllllll}
\hline Case & & $\begin{array}{l}\text { Pressure } \\
\text { in MPa }\end{array}$ & $\begin{array}{l}\text { Velocity } \\
\text { in } \mathrm{m} / \mathrm{s}\end{array}$ & $\begin{array}{l}\mathrm{T} 1 \\
\text { in K }\end{array}$ & $\begin{array}{l}\mathrm{Ta}_{i n j} \\
\text { inK }\end{array}$ & $\begin{array}{l}\mathrm{Tb}_{\text {inj }} \\
\text { in K }\end{array}$ \\
\hline 3 & (RCM1A) & 3.97 & 4.9 & 120.9 & 126.9 & 122.2 \\
4 & & 3.98 & 5.4 & 130.7 & 137.0 & 133.3 \\
5 & 4.90 & 2.0 & 120.7 & 131.0 & 122.6 \\
6 & 4.90 & 1.9 & 129.8 & 140.0 & 131.5 \\
7 & 5.01 & 4.5 & 120.7 & 126.2 & 122.5 \\
8 & 5.00 & 4.9 & 130.2 & 135.7 & 131.7 \\
9 & 5.85 & 2.0 & 120.7 & 135.0 & 122.8 \\
10 & & 5.88 & 1.9 & 129.9 & 140.5 & 131.9 \\
11 & (RCM1B) & 5.98 & 4.9 & 121.7 & 128.7 & 123.3 \\
\hline
\end{tabular}

\section{II.B. Key Challenge: Temperature}

The main challenge remains to accurately determine the fluid temperature. Figure 1(a) illustrates how sensitive the density is in regard to the temperature for two of the pressure levels of the experiment. Especially for temperatures between $120 \mathrm{~K}$ and $140 \mathrm{~K}$, even minor changes / inaccuracies of a few Kelvin can easily yield differences in density by a factor of two. Despite the sophisticated experimental approach, inaccuracies remain: measurements of injectant temperature at the injector exit differ up to $8 \mathrm{~K}$ for the same experiment 
(Tab. 1), depending on the orientation of the thermo couple alone. The authors suggest entrainment of ambient temperature gas to the sensor for the case of the perpendicularly oriented thermo couple, thus yielding a higher temperature than for the axial measurement.

While injecting cryogenic nitrogen until the injector is cooled down to the nominal temperature before beginning the measurements might be a good procedure to ensure the desired temperature, it raises the question of timing. With no information about the time it took for the injector to cool down, it is unknown how much cryogenic nitrogen has entered the initially ambient temperature chamber. The flow field surrounding the free jet hence changes in time and its exact state at the time of the Raman measurements or temperature measurements is unknown.

\section{II.C. Numerical Approaches}

\section{II.C.1. The RCM1 Test Cases}

The RCM1 test cases have been defined for the 2001 Rocket Combustion Modeling workshop as CFD test cases $^{6}$ based on Mayer's cases 3 and 11. The test cases have been investigated by other researchers since. Boundary conditions have been defined to be used by the participants. As the cylinder wall is heated, it is isothermal at $297 \mathrm{~K}$, faceplate and injector are assumed adiabatic.

Mayer et al. ${ }^{11}$ and Branam et al. ${ }^{3}$ themselves carried out CFD computations. They used the CFD-ACE

Table 2. RCM1 numerical boundary conditions ${ }^{6}$

\begin{tabular}{rl|ll} 
& & RCM1A & RCM1B \\
\hline Chamber pressure in & $\mathrm{MPa}$ & 3.97 & 5.98 \\
Temperature in & $\mathrm{K}$ & 126.9 & 128.7 \\
Density in & $\mathrm{kg} / \mathrm{m}^{3}$ & 457.5 & 514.0 \\
Mass flow in & $\mathrm{kg} / \mathrm{s}$ & 0.00995 & 0.01069 \\
Velocity in & $\mathrm{m} / \mathrm{s}$ & 0.769 & 0.736
\end{tabular}

incompressible Navier-Stokes solver, extended for treatment of high pressure nitrogen. A k- $\epsilon$ turbulence model has been used. CFD results, as displayed in Fig. 2(b), show a reasonable agreement with experiments for axial density profiles from approximately 10 injector diameters downstream of the injector exit. A striking

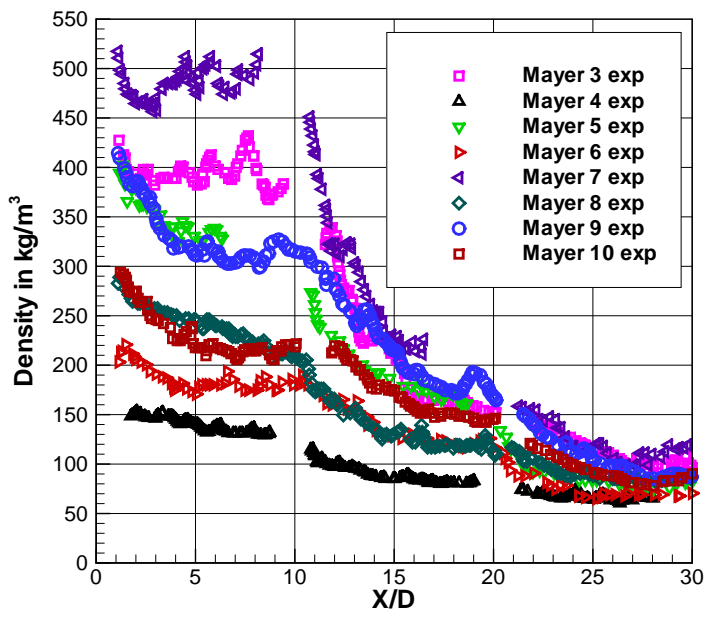

(a) Measured axial densities.

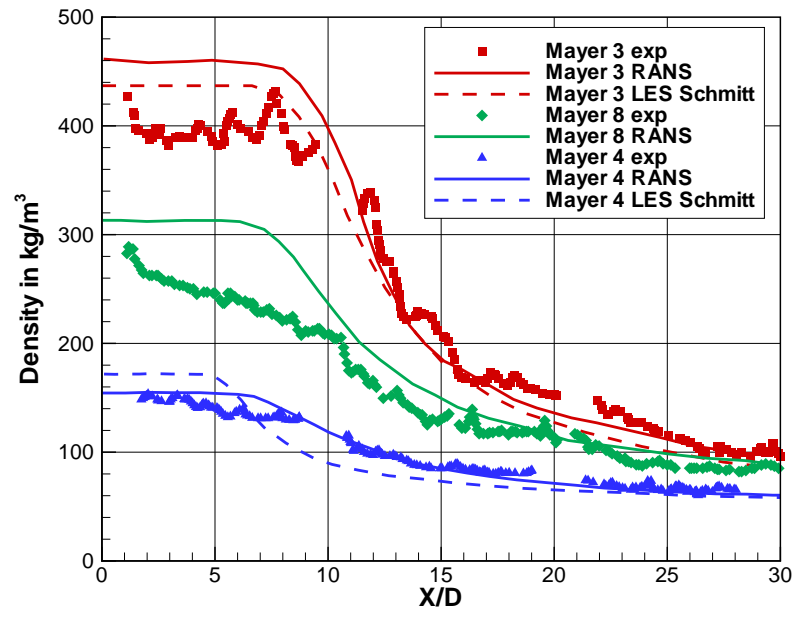

(b) Comparison of experiment and CFD.

Figure 2. Axial density profiles.

feature shared by all shown CFD results, however, is the presence of a stable dense core protruding into the chamber. However, upon reviewing Fig. 2(a), it can be seen that experiments rarely show a region of constant density at the injector exit. in fact, of the shown eight density profiles, only cases 7 and 4 exhibit 
a region of constant density. All other cases sport a rapid reduction in density from the injector exit. The question hence is why the chosen method of Mayer and Branam showed this particular behavior, and whether this is a general shortcoming of the chosen method or something solvable for our future work with similar models. Possible faulty assumptions/methods are

1. 2D-axisymmetry

2. Steady flow field assumption

3. Turbulence models

4. Boundary Conditions

In order to accelerate computations, the flowfield inside the cylindrical chamber is assumed to be axisymmetric, i.e. a two-dimensional cut completely describes the problem. However, numerical investigations of Hosangadi et al. ${ }^{7}$ show a helical instability for a cryogenic nitrogen injection with co-flow, hence this assumption might not be valid. Clearly, in presence of three-dimensional instabilities, the flow is not steady. Originally, turbulence models such as $\mathrm{k}-\epsilon$ have been derived and calibrated for incompressible flow. Compressibility corrections have been introduced by Wilcox. However, these are triggered by Mach number instead of density gradients. It is possible that an expanding cryogenic nitrogen jet, which drastically increases its specific volume, is not properly modelled by conventional turbulence models. Finally, the inflow boundary conditions have much influence on the structure of a free jet.

A literature review shows that LES computations show a behavior similar to Mayer's results: Schmitt et al. ${ }^{15}$ computed cases 3 and 4 of Mayer's experiments and predicted a stable, dense core which is not seen in experimental data for case 4 (Fig. 2(b)). This strongly suggests that assumptions 1 to 3 are not the sole cause of the dense core issue, as the computations have been unsteady, 3D, and using a more detailed treatment of turbulence. If one includes cryogenic nitrogen injection cases other than Mayer's experiments, one finds yet other researchers, either using a RANS approach (Barata et al. ${ }^{2}$ ) or LES (Zong et al. ${ }^{16}$ ) that again predict a stable, dense core, extending approximately 10 injector diameters downstream from the injector exit.

We therefore suggest that the cause should be sought in the proper definition of boundary conditions.

\section{II.C.2. Physical Boundary Conditions vs. Numerical Boundary Conditions}

In order to check hypothesis four of the preceding section, the boundary conditions of the published CFD studies have to be analyzed. The methods differ in including the injector into the computation, thus computing the inflow profile (Mayer) or imposing a turbulent velocity field (Schmitt) or velocity and temperature top-hat profiles (Barata). All aforementioned CFD studies have in common that the injectant features a top-hat temperature profile when it enters the chamber. Due to the adiabatic walls, only viscous effects will act to change the profile, however, this effect is rather weak.

Closely reading Mayer's paper makes clear that heat transfer in the injector is not negligible. Mayer writes "These results indicate, first of all, the heat transfer in the injection tube is significant, particularly in the warmer cases, affecting the length of the potential core and dissipation of the jet." Radial density profiles show a distinct soft slope already 1.2 injector diameters downstream (Fig. 6(a)). From the description of the experiment it is clear that the exact state of the injector could not be determined during the experiments. However, the temperature of the injectant changes substantially while passing through the injector, as can be seen in Table 1: The temperatures at the injector exit $\left(\mathrm{Ta}_{i n j}, \mathrm{~Tb}_{i n j}\right)$ are higher than upon entering the injector (T1).

Therefore it seems reasonable to perform computations taking into account heat transfer from the wall to the fluid inside the injector.

\section{Computations}

\section{III.A. The DLR TAU Code}

Computations are carried out using the DLR TAU Code. ${ }^{5,10}$ TAU is a hybrid grid, Godunov-type finite volume CFD code which solves the integral form of the Euler and Navier-Stokes equations. TAU achieves second order accuracy in space using MUSCL reconstruction. It has been verified for a variety of steady and unsteady flow cases, covering sub-, trans-, super-, and hypersonic Mach numbers. TAU has been extended 
by Bartolome Calvo et al. ${ }^{4}$ to deal with pure substance real gas equations of state. Real gas properties are calculated in a preprocessing step and stored in lookup-tables in order to improve numerical efficiency. Here, the Modified Benedict Webb Rubin (MBWR) EOS ${ }^{9}$ has been employed to determine nitrogen real gas properties. A Spalart Allmaras turbulence model is employed.

\section{III.B. Computational Domain and Boundary Conditions}

As a first testcase, the injection of cryogenic nitrogen into an environment at supercritical pressure is computed. The test case is defined as case 11 by Mayer at al., ${ }^{11}$ corresponding to RCM1B. Injection condition is a temperature of $128 \mathrm{~K}$, a density of $514 \mathrm{~kg} / \mathrm{m}^{3}$ with a corresponding pressure of $5.98 \mathrm{MPa}$. This point is marked in Fig. 1(a). Clearly, the fluid cannot be regarded as an ideal gas at this thermodynamic state.

The computational domain encompasses the $2.2 \mathrm{~mm}$ diameter, $90 \mathrm{~mm}$ length injector, attached to a chamber, as used in the experiments. The chamber geometry and boundary conditions, however, are chosen to not conform to experiments. As discussed in Section II.B, the exact time of the density measurements and thus state of the fluid inside the chamber is unknown. The experiment up to this point is thus unsteady. While a steady state is imaginable, i.e. when the chamber is completely flooded by cryogenic nitrogen, only heated up at the chamber walls, radial density plots exhibit a rapid drop off of density towards room temperature values (Fig.6(a)). This suggests that the steady state has not been reached at the point of the Raman measurements.

In order to have a defined steady state where the cryogenic nitrogen enters room temperature surroundings, the numerical setup is chosen to differ from the experimental setup: instead of computing a closed chamber that slowly floods with dense nitrogen and evaluating this at an arbitrary point in time, an open surrounding is modelled, as depicted in Fig. 3(a). The faceplate remains a viscous wall. The outer wall boundary condition is replaced with a farfield boundary condition. The distance from the injector $(27 \mathrm{D}$ in radial direction, $181 \mathrm{D}$ in axial direction) are well beyond the $6 \mathrm{D}$ radial and $40 \mathrm{D}$ axial distance specified by Zong et al. ${ }^{16}$ as being sufficiently far away to minimize boundary influence on the free jet. The structured grid is comprised of 220,000 cells, a detail of the injector is shown in Fig. 3(b).

Injector wall boundary conditions have been set either adiabatic or sothermal, as already discussed.

All inflow properties are imposed as block profile Dirichlet boundary conditions at the beginning of the injector.

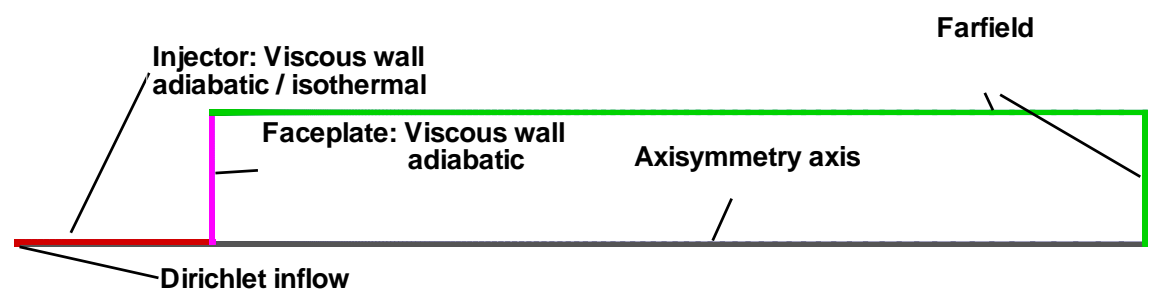

(a) Boundary conditions.

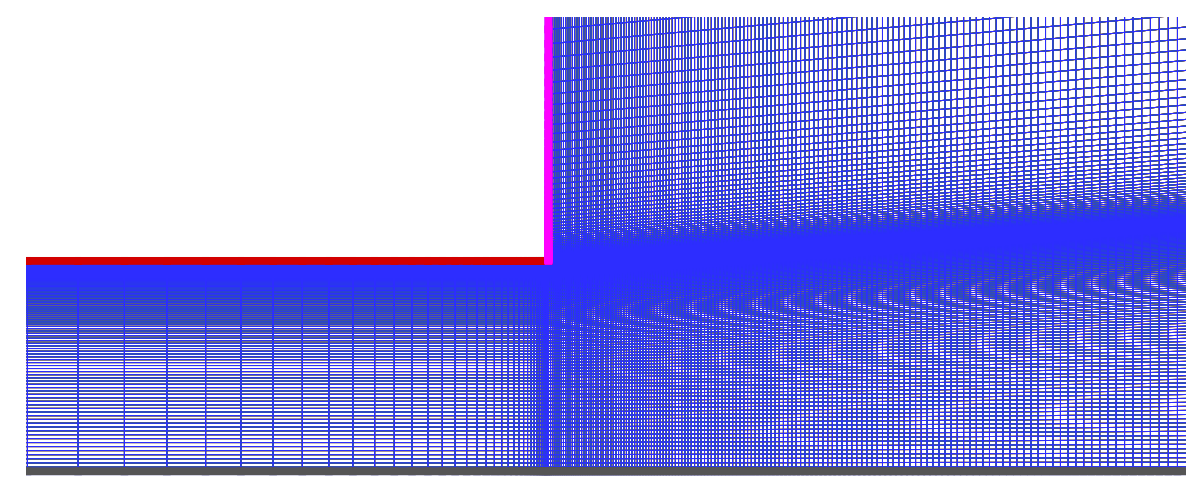

(b) Grid near injector.

Figure 3. Computational domain 


\section{Results}

\section{IV.A. Adiabatic Wall}

The first case features the classical adiabatic wall injector configuration. Temperature and density contours are shown in Fig. IV.A.

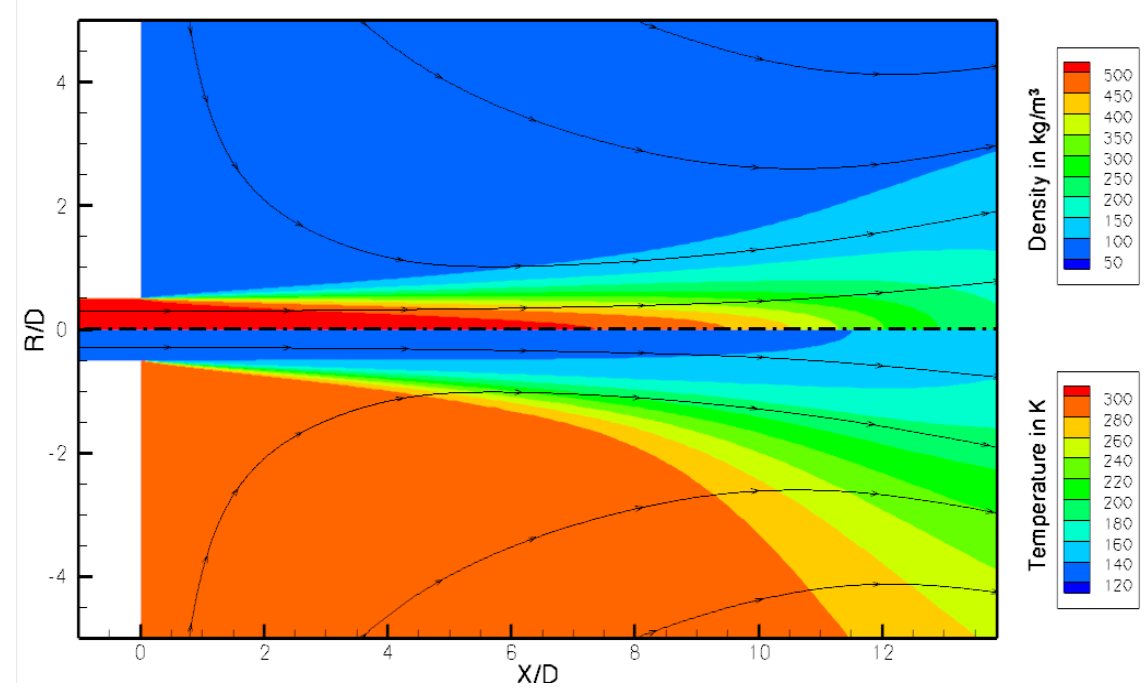

Figure 4. Density and temperature field of dense nitrogen injection into nitrogen environment.

The jet spreads laterally, as can be seen from the streamlines, as one would expect from a classical free jet. However, the peculiar nature of the transcritical jet can be seen when the range of the variables is regarded. Following the temperature plot, the cryogenic jet emanates from the injector at $128 \mathrm{~K}$ and $514 \mathrm{~kg} / \mathrm{m}^{3}$. Surrounding flow at $297 \mathrm{~K}$ and $68 \mathrm{~kg} / \mathrm{m}^{3}$ is entrained and heats the free stream. A dense axial core can be seen which extends to approximately $\mathrm{X} / \mathrm{D}=10$ before the density drops off quickly. This is in accordance with other CFD studies, as discussed above. As typical for cryogenic jets, a moderate change in temperature is accompanied by a drastic change in density. E.g. at $\mathrm{X} / \mathrm{D}=15$, the temperature has risen by $20 \%$ to $158 \mathrm{~K}$ while the density has dropped to almost a third, $182 \mathrm{~kg} / \mathrm{m}^{3}$.

\section{IV.B. Heated Wall}

As this dense core is typically not seen in experiments, we carried out another computation. Instead of adiabatic injector walls, now the wall temperature is set to $298 \mathrm{~K}$, i.e. ambient conditions, as can be expected at the beginning of the experiment. The change of the near injector flow field is substantial, as can be seen in Fig. IV.B. A lower density boundary layer develops at the heated wall. Figure 6(a) compares density profiles in the injector exit and immediately downstream of it. The adiabatic temperature profile is nearly top hat, only slightly changed by viscous effects. At $\mathrm{D} / \mathrm{X}=1.2$, the profile is already distorted. The isothermal wall temperature profile behaves different. A developed profile can be seen, featuring a smooth transition between ambient density and the centerline density. The centerline density is already reduced slightly compared to the adiabatic case. Due to lack of gradients, the profile is almost unchanged at $\mathrm{D} / \mathrm{X}=1.2$. The experimental densities are higher than both cases, in fact, they are higher than the inlet density described for the RCM1B test case. However, the profile clearly resembles the computation taking into account wall heat transfer.

Figure 6(b) shows the axial density distribution. The desired density drop off without dense core can be seen. The temperature at the injector axis starts to drop at $\mathrm{X} / \mathrm{D}=-5$. In the chamber, the jet expands less and overall reduces its density faster. As only radial profiles have been published of the RCM1B case, only three experimental results are known. While the measured density closest to the injector axis is higher than in both computations, the wall heat transfer case is closer to the other known data points. 


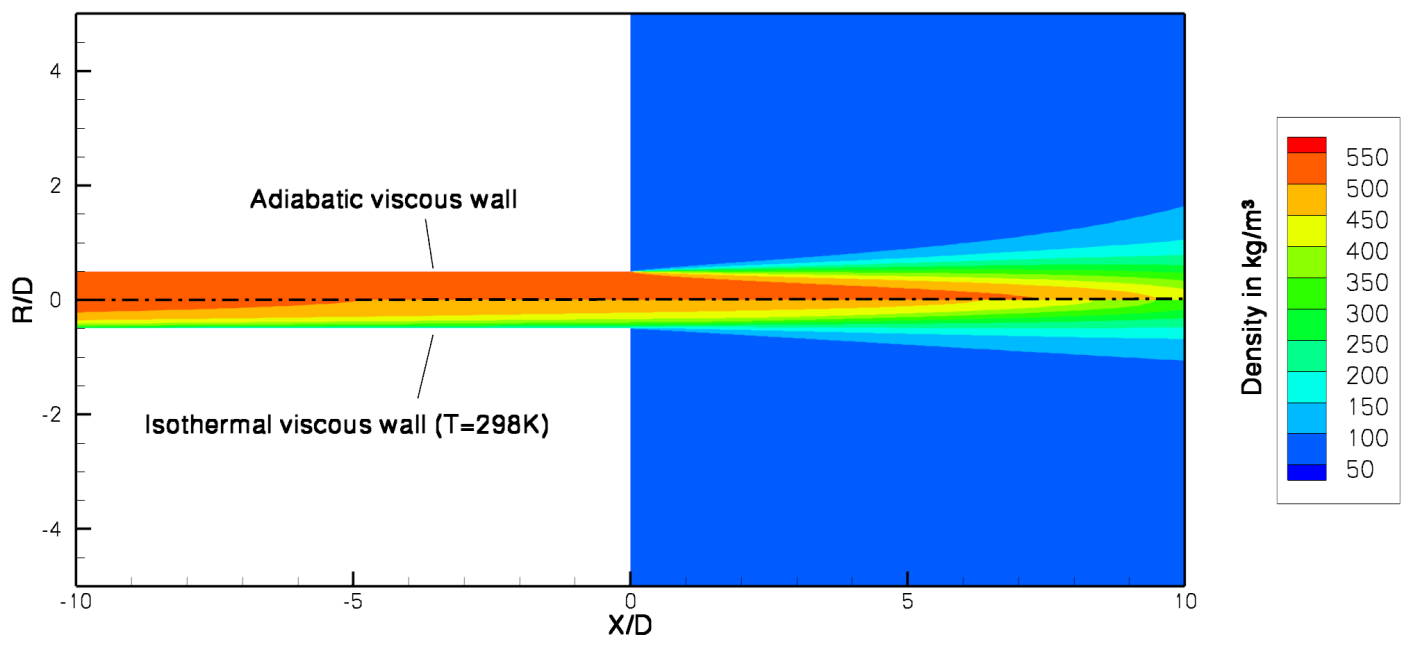

Figure 5. Comparison of density fields for adiabatic wall case (top) and isothermal wall case (bottom).

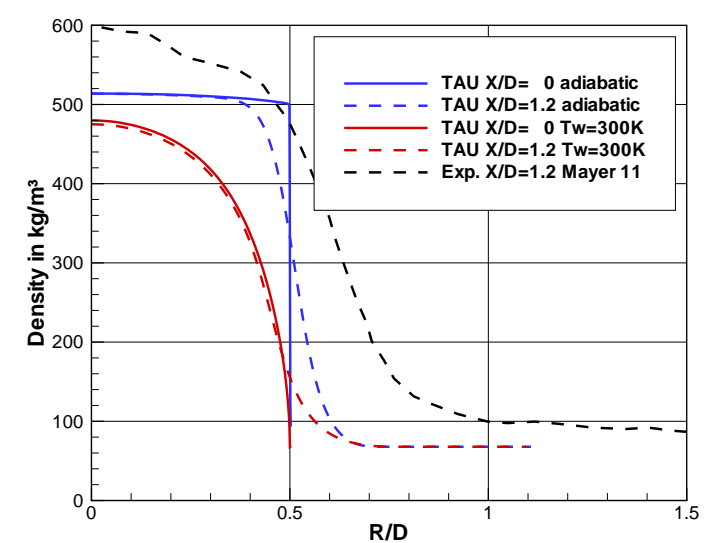

(a) Radial density profiles at injector exit and near field

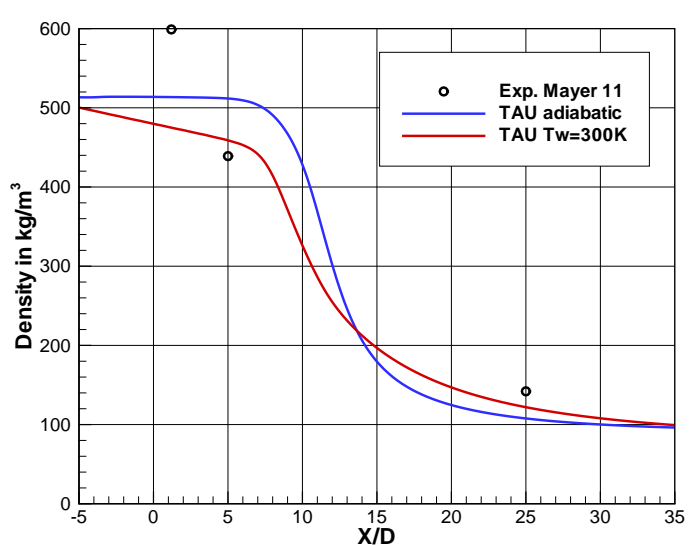

(b) Axial density distribution

Figure 6. Comparison of density profiles for adiabatic and isothermal injector wall.

\section{Conclusion and Outlook}

This paper reported on a first step of a continuous phase propellant injection model for liquid rocket engines. Experiments have been discussed that show a continuous behavior of injected propellant. A continuous description of the fluid, i.e. in a Eulerian manner thus is a physically reasonable approach. Key component is a real gas equation of state to accurately model fluid behavior near the critical pressure, as deviations from ideal gas behavior is substantial.

An injection experiment has been analyzed. It has been found that a 'liquid core' cannot be seen in most experiments.

Furthermore, it has been discussed that heat transfer inside the injector from the wall to the fluid is nonnegligible in experiments.

A review of CFD studies has shown that state of the art methods predict a dense core, despite different levels of sophistication (incompressible RANS, compressible FANS, LES). The common feature is the assumption of a temperature block profile in the injector upon entering the chamber.

Taking into account wall heat flux in CFD suppresses the dense core and introduces a behavior better fitting experimental observations. 
This suggests that the atomization process of a cryogenic jet at supercritical pressures is more of thermodynamic nature, i.e. pseudo boiling, than a mechanical process like liquid jet atomization.

\section{References}

${ }^{1}$ D.T. Banuti and K. Hannemann. Interfacial area transport equation in Statistical-Eulerian-Eulerian simulations of multiphase flow. In A. Dillmann, G.Heller, M.Klaas, H.-P. Kreplin, W.Nitsche, and W. Schrder, editors, New Results in Numerical and Experimental Fluid Mechanics VII, volume 112 of Notes on Numerical Fluid Mechanics and Multidisciplinary Design (NNFM). Springer, 2010.

${ }^{2}$ J.M.M. Barata, I. Gökalp, and A.R.R. Silva. Numerical study of cryogenic jets under supercritical conditions. Journal of Propulsion and Power, 19(1):142-147, 2003.

${ }^{3}$ R. Branam and W. Mayer. Characterization of cryogenic injection at supercritical pressure. Journal of Propulsion and Power, 19(3):342-355, 2003.

${ }^{4} \mathrm{~J}$. Bartolome Calvo and K. Hannemann. Numerical simulation of liquid rocket engine cooling channels. In Proceedings of the 45th AIAA/ASME/SAE/ASEE Joint Propulsion Conference \&S Exhibit, Denver, 2009.

${ }^{5}$ T. Gerhold, M. Galle, O. Friedrich, and J. Evans. Calculation of complex three-dimensional configurations employing the DLR-TAU-code. In Proceedings of the 35th Aerospace Sciences Meeting and Exhibit, number AIAA-1997-167, Reno, USA, 1997. AIAA.

${ }^{6}$ Oskar J Haidn. Proceedings of the second international workshop rocket combustion modeling - atomization, combustion and heat transfer. Lampoldshausen, 2001.

${ }^{7}$ A. Hosangadi, C.P. Lee, C. Kannepalli, and S. Arunajatesan. Three-dimensional hybrid rans/les simulations of a supercriticcal liquid nitogen jet. In Proceedings of the 44th AIAA/ASME/SAE/ASEE Joint Propulsion Conference 85 Exhibit, Hartford, 2008.

${ }^{8} \mathrm{~S}$. Jay, F. Lacas, and S. Candel. Combined surface density concepts for dense spray combustion. Combustion and Flame, 144:558-577, 2006

${ }^{9}$ K. K. Kuo. Principles of Combustion (2nd Edition). Wiley, 2005.

${ }^{10}$ A. Mack and V. Hannemann. Validation of the unstructured DLR-TAU-code for hypersonic flows. In Proceedings of the 32nd AIAA Fluid Dynamics Conference and Exhibit, number AIAA-2002-3111, 2002.

${ }^{11}$ W. Mayer, J. Telaar, R. Branam, G. Schneider, and J. Hussong. Raman measurements of cryogenic injection at supercritical pressure. Heat and Mass Transfer, 39, 2003.

${ }^{12}$ M. Oschwald, J.J. Smith, R. Branam, J. Hussong, A. Schik, B. Chehroudi, and D. Talley. Injection of fluids into supercritical environments. Combustion Science and Technology, 178:49-100, 2006.

${ }^{13}$ H. Palliere, C. Corre, and J. R. G. Cascales. On the extension of the AUSM+ scheme to compressible two-fluid models. Computers \&s Fluids, 32:891-916, 2003.

${ }^{14}$ D. Preclik, O. Knab, J. Görgen, and G. Hagemann. Progress in Astronautics and Aeronautics: Liquid Rocket Thrust Chambers: Aspects of Modeling, Analysis, and Design, chapter Simulation and Analysis of Thrust Chamber Flowfields: Cryogenic Propellant Rockets. Number ISBN-13: 978-1563472237. AIAA, 2004.

${ }^{15}$ T. Schmitt, L. Selle, B. Cuenot, and T. Poinsot. Large-eddie simulation of transcritical flows. Comptes Rendus Mecanique, 337:528-538, 2009.

${ }^{16} \mathrm{~N}$. Zong, H. Meng, and V. Yang. Numerical study of cryogenic fluid injection and mixing under supercritical conditions. Physics of Fluids, 16(12):4248-4261, 2004. 